\title{
Blood group related antigens in ocular cicatricial pemphigoid
}

\author{
C Creuzot-Garcher, T H-Xuan, A M Bron, H Robin, P d'Athis, J Bara
}

Br J Ophthalmol 2004;88:1247-1251. doi: 10.1136/bjo.2003.039784

Aim: To study the MUC5AC and the blood group related antigen expression in ocular cicatricial pemphigoid (OCP) according to the distribution of Lewis and secretor phenotypes in OCP patients compared to normal subjects.

Methods: Immunostaining was performed on conjunctival biopsy specimens from 22 consecutive patients suffering from $\mathrm{OCP}$, using monoclonal antibodies (Mabs) directed against the peptidic core MUC5AC mucin (anti-M1/MUC5AC Mabs) and against the saccharide moieties (anti-blood group related antigens). These latter included anti-Le ${ }^{a}$, anti-Le ${ }^{b}$, anti-sialyl Le ${ }^{a}$, and $\mathrm{H}$ type 2 Mabs, which immunoreact with Lewis positive and non-secretor (Le $\left.{ }^{a}\right)$, Lewis positive and secretor $\left(L^{b}\right)$, Lewis positive (sialyl Le ${ }^{a}$ ), and secretor (H type 2) phenotypes respectively. Serological tests were also performed to confirm the phenotype of each patient. The immunohistopathological patterns and the distribution of Lewis and secretor phenotypes were compared with the results of a previous study in normal individuals.

Results: (1) In OCP patients compared to the normal population, anti-MI immunoreactivity of goblet cells was unchanged, whereas anti-Le ${ }^{a}$, anti-Le ${ }^{b}$, and anti-sialyl Le ${ }^{a}$ immunoreactivities of epithelial and/or goblet cells were markedly decreased. (2) $41 \%$ of OCP patients had a nonsecretor phenotype, which is statistically significantly more than the estimated incidence of the same phenotype in the French population $(20 \%)(p \approx 0.04)$.

Conclusions: Mucins in OCP patients showed a decreased expression of blood group related antigens whereas the MUC5AC peptidic core detected by anti-M1 Mab remained unchanged. These results also seem to indicate that OCP may be associated with a non-secretor phenotype.

\footnotetext{
C
} icatricial pemphigoid (CP) is a rare systemic mucocutaneous bullous autoimmune disease which affects the eye in $70 \%$ of cases. ${ }^{1-3}$ Ocular cicatricial pemphigoid (OCP) is characterised by chronic conjunctivitis, progressive subepithelial fibrosis, fornix foreshortening, symblepharon formation, and dry eye syndrome. Immune deposits at the conjunctival epithelial basement membrane zone are the hallmark of OCP. ${ }^{4-6}$

Mucus is a tear film component which is crucial for the adherence of the aqueous layer to the superficial epithelial cells of the ocular surface. Mucins are made of flexible threads containing subunits. ${ }^{7}$ These latter contain heavily glycosylated regions ( $\mathrm{T}$ domains) interspersed with less glycosylated or "naked" stretches of proteins. ${ }^{8}$ Carbohydrate moieties are important components of mucus partly responsible for its viscosity. The blood group related epitopes are well known mucin antigens, mainly located in the glycosylated $\mathrm{T}$ domains. $\mathrm{Le}^{\mathrm{a}}$ and $\mathrm{Le}^{\mathrm{b}}$ antigenic determinants result from the epistatic interaction of the products of Lewis (Le or
FUT3/5) and Secretor (Se or FUT2) genes on the Le ${ }^{\mathrm{c}}$ precursor chain. ${ }^{8}$ These genes encode for two different fucosyltransferases which each transfers one fucose molecule onto two distinct sites of the $\mathrm{Le}^{\mathrm{c}}$ chain to build $\mathrm{Le}^{\mathrm{a}}$ and $\mathrm{Le}^{\mathrm{d}}$ epitopes. The transfer of two fucose molecules on the $\mathrm{Le}^{\mathrm{c}}$ chain by both fucosyltransferases builds the $\mathrm{Le}^{\mathrm{b}}$ antigen. Four different phenotypes-namely, $\mathrm{Le}^{\mathrm{a}}, \mathrm{Le}^{\mathrm{b}}, \mathrm{Le}^{\mathrm{c}}$ and $\mathrm{Le}^{\mathrm{d}}$-are thus generated depending on the enzyme activities of these two fucosyltransferases. ${ }^{9}$ The links between Lewis phenotypes and $L e$ and $\mathrm{Se}$ genes are detailed in table 1.

Mucin Ml/MUC5AC peptidic core epitopes, antigens secreted by conjunctival goblet cells, are mainly found in the columnar mucus cells of the gastric surface epithelium. ${ }^{10}{ }^{11}$ All these epitopes probably are encoded by the same MUC5AC gene. ${ }^{12-14}$

In dry eye syndrome, mucin distribution and biochemical characteristics, including glycosylation, are modified. ${ }^{15}$ However, studies using conventional histological stainings ${ }^{16}$ or lectins ${ }^{17}$ have failed to show any alteration of carbohydrates in the goblet cells of patients with OCP, which is usually associated with dryness.

The purpose of our study was to immunocharacterise the peptidic antigens of conjunctival mucins and to analyse the distribution of the blood group related antigens with respect to the secretor and Lewis phenotypes in OCP patients.

\section{PATIENTS AND METHODS \\ Population study}

Twenty two patients including 16 biopsy proved OCP patients and six OCP suspects, with a mean age of 65 years (range 2692 years) were included in the study. The diagnosis of OCP suspect relied on the combination of the following criteria: age over 60, progressive conjunctival fibrosis, association with extraocular involvement, absence of prolonged use of preserved topical medications, absence of systemic and ocular non-autoimmune fibrosing conditions including StevensJohnson syndrome, toxic epidermal necrolysis, Sjögren's syndrome, sarcoidosis, lupus, progressive systemic sclerosis, fibrosing infectious conjunctivitis, ocular trauma or burn, rosacea, atopic keratoconjunctivitis, and efficacy of systemic immunosuppressive therapy.

Eighty nine normal individuals who belonged to a previous study were also included. ${ }^{18}$ A conjunctival biopsy had been performed during cataract surgery, and the specimens had been processed with the same methods as in this study.

\section{Tissue samples}

Conjunctival biopsies were harvested from the bulbar conjunctiva adjacent to the superior limbus after informed consent had been obtained. They were bisected or trisected,

Abbreviations: OCP, ocular cicatricial pemphigoid; Mabs, monoclonal antibodies 
Table 1 Lewis phenotypes

\begin{tabular}{|c|c|c|c|c|}
\hline Name of phenotype & $\operatorname{Le}^{a}$ & $L e^{b}$ & $L e^{c}$ & $L e^{d}$ \\
\hline Structures & 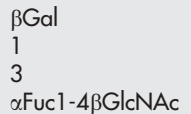 & 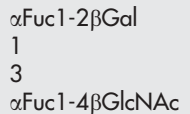 & $\begin{array}{l}\beta \text { Gal } \\
1 \\
3 \\
\beta G \mid c N A c\end{array}$ & $\begin{array}{l}\alpha \text { Fucl-2 } 2 \beta \text { Gal } \\
1 \\
3 \\
\beta G l_{c N A c}\end{array}$ \\
\hline Secretor(FUT2) & - & + & - & + \\
\hline Lewis (FUT3/5) & + & + & - & - \\
\hline Red cell phenotype & $\operatorname{Le}(a+b-)$ & $\operatorname{Le}(a-b+)$ & $\operatorname{Le}(a-b-)$ & Le $(a-b-)$ \\
\hline $\begin{array}{l}\text { Frequency in white people (\%) } \\
\text { MAbs reactivity }\end{array}$ & 20 & 70 & 1 & 9 \\
\hline Anti-Le & +r+ & - & - & - \\
\hline Anti-Le ${ }^{b}$ & $t^{*}$ & +r+ & - & - \\
\hline Anti-S Le ${ }^{a}$ & ++ & ++ & - & - \\
\hline Anti-H-Type 2 & - & H+ & - & +++ \\
\hline
\end{tabular}

*Anti-Le ${ }^{b}$ MAbs faintly react with Le ${ }^{a}$ antigen. ${ }^{?}$

Lewis positive (Le $(a+b-)$ and Le $(a-b+))$ patients can be detected by anti-Le ${ }^{a}$, anti-Le ${ }^{b}$, and/or anti-sialyl Le ${ }^{a}$ Mabs. Non-secretor patients include those with Le ${ }^{a}$ phenotype (detected by anti-Le ${ }^{a}$ Mabs) and Le ${ }^{c}$ phenotype which fails to immunoreact with any anti-Lewis and anti-H type 2 Mabs. Secretor patients are detected by anti-Le but also by anti-H type 2 Mabs.

with one or two pieces processed for direct immunofluorescence and/or direct immunoelectron microscopy, and another piece for immunocharacterisation of mucin epitopes.

This latter piece was fixed in 95\% ethanol overnight, as previously described, ${ }^{16}$ embedded in paraffin, and then thin serial sections were cut.

\section{Monoclonal antibodies}

All Mabs except for one (Mab NS-19-9) were provided by our laboratory. They included anti-Ml Mabs, ${ }^{11-14}$ and the Mabs against the saccharide moieties of mucins. These latter included anti-Le ${ }^{\mathrm{a}}$ (7LE), ${ }^{19-21}$ anti-Le ${ }^{\mathrm{b}}$ (2-25LE), ${ }^{22}$ anti-H type 2 (19-0LE), ${ }^{19-21}$ and anti-sialyl Le ${ }^{\mathrm{a}}$ (NS 19-9) ${ }^{23}$ Mabs. Anti-Le ${ }^{\mathrm{b}}$ Mabs are known to faintly cross react with the Le ${ }^{\mathrm{a}}$ antigen (tables 1 and 2). ${ }^{9}$

The links between the blood group antigen related structures, secretor and Lewis phenotypes and Le and Se genes are detailed in table 1 . Lewis positive patients can be detected by anti-Le ${ }^{\mathrm{a}}$, anti-Le ${ }^{\mathrm{b}}$, and anti-sialyl Le ${ }^{\mathrm{a}}$ Mabs. Nonsecretor patients include those with $\mathrm{Le}^{\mathrm{a}}$ phenotype (detected by anti-Le $e^{a}$ Mabs) and $\mathrm{Le}^{\mathrm{c}}$ phenotype which fails to immunoreact with any anti-Lewis Mab. Since the fucosyltransferase encoded by Se gene also transfers fucose onto a precursor chain different from $\mathrm{Le}^{\mathrm{c}}$ chain to build the $\mathrm{H}$ type 2 antigen, ${ }^{10}$ secretor patients not only are detected by anti-Le ${ }^{\mathrm{b}}$, but also by anti-H type 2 Mabs (table 1).

\section{Immunoperoxidase assay}

The sections were deparaffinised and incubated for $30 \mathrm{~min}-$ utes with the Mabs (undiluted hybridoma supernatants).
After three washes in PBS-Tween, the sections were incubated for 30 minutes with sheep anti-mouse Ig antibodies $(1 / 200)$ bound to peroxidase. After three washes in PBS-Tween, the sections were incubated for 4 minutes with amino-ethylcarbazole containing $\mathrm{H}_{2} \mathrm{O}_{2}$. Cell nuclei were then stained with $1 \%$ haematein. To control the specificity of the immunoreactivity, Mabs were absorbed with gastric Ml mucin preparation $(100 \mu \mathrm{g} / \mathrm{ml})$. Semiquantitative grading of the intensity of immunostaining from 0 to $3+$ was performed in a masked fashion using light microscopy. Gastroduodenal mucosae of individuals expressing each Lewis and secretor phenotype were used as positive controls as previously described. ${ }^{10}$

\section{Serological determination of Lewis phenotypes}

Serological Lewis phenotypic determination, obtained using haemagglutination after informed consent of the patients had been obtained, was compared to the phenotypes determined by immunohistochemistry of the conjunctiva.

\section{Statistics}

Phenotypic distribution was studied using the Fisher's exact test. Results were considered as statistically significant when the $\mathrm{p}$ value was less than 0.05 .

\section{RESULTS}

\section{Mucin M1/MUC5AC peptidic core epitopes}

\section{Goblet cells}

The number of conjunctival goblet cells was dramatically reduced in OCP patients compared to normal controls. In

Table 2 Immunohistological staining using anti-blood group related antigen Mabs on the conjunctiva of the 22 OCP patients and the 89 normal individuals ${ }^{18}$

\begin{tabular}{|c|c|c|c|c|c|c|c|c|c|c|c|}
\hline & \multirow[b]{3}{*}{$\mathrm{Ph}$} & \multirow[b]{3}{*}{ No* } & \multirow[b]{3}{*}{$\%$} & \multicolumn{4}{|c|}{ Intensity of staining } & \multicolumn{4}{|c|}{ Intensity of staining } \\
\hline & & & & \multicolumn{4}{|c|}{ Epithelial cells } & \multicolumn{4}{|c|}{ Goblet cells } \\
\hline & & & & $\overline{L e^{a}}$ & $S-L^{a}$ & $L e^{b}$ & H-type 2 & $\overline{L^{a}}$ & $S-\mathrm{Le}^{a}$ & $L e^{b}$ & H-type2 \\
\hline $\mathrm{N}$ & $\mathrm{Le}^{\mathrm{a}}$ & 17 & 20 & +++ & +++ & + & - & +++ & +++ & + & $\begin{array}{ll}- \\
-\end{array}$ \\
\hline OCP & $\mathrm{Le}^{\mathrm{a}}$ & 7 & 32 & +/++ & $+/++$ & $+1-$ & - & $+/-\dagger$ & $+/-\dagger$ & $+1-\dagger$ & $-\dagger$ \\
\hline $\mathrm{N}$ & $\mathrm{Le}^{\mathrm{b}}$ & 63 & 70 & - & +++ & +++ & +H+ & +H+ & +H+ & + & + \\
\hline OCP & $\mathrm{Le}^{\mathrm{b}}$ & 13 & 59 & - & $+1-$ & $+/++$ & ++ & $+/-\ddagger$ & $+/-\ddagger$ & $+/-\ddagger$ & $+\ddagger$ \\
\hline $\mathrm{N}$ & $\mathrm{Le}^{\mathrm{c}}$ & 0 & 0 & NA & NA & NA & NA & NA & NA & NA & NA \\
\hline OCP & $\mathrm{Le}^{\mathrm{c}}$ & 2 & 9 & - & - & - & - & - & - & - & - \\
\hline $\mathrm{N}$ & $\operatorname{Le}^{d}$ & 9 & 10 & - & - & - & ++ & - & - & - & ++ \\
\hline OCP & $\operatorname{Le}^{d}$ & 0 & 0 & NA & NA & NA & NA & NA & NA & NA & NA \\
\hline
\end{tabular}

OCP, ocular cicatricial pemphigoid; N, normal conjunctiva; Ph, phenotype; Lea, anti-Le ${ }^{a}$ (7LE); S-Le ${ }^{a}$, anti-sialyl-Le ${ }^{a}$ (NS 19-9); Le , anti-Le $^{b}$ (2-25 LE); positive reaction from + to ++ represent increasing number of stained cells and intensity staining; NA, not applicable; -, negative staining; "number of individuals; tgoblet cells were found only in $5 / 7$ Le ${ }^{a}$ OCP patients; tgoblet cells were found only in $11 / 13$ Le ${ }^{b}$ OCP patients. 


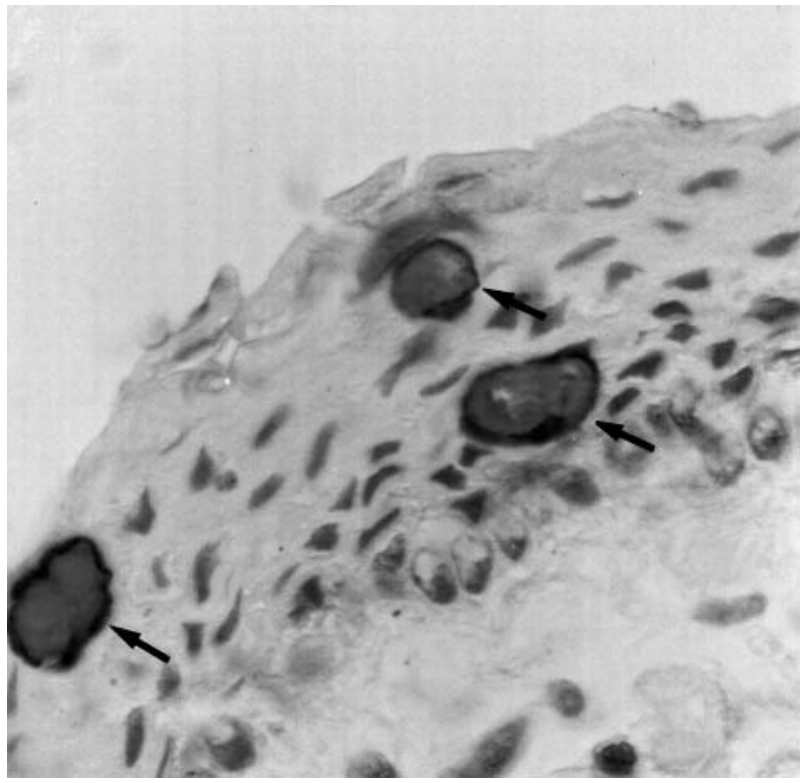

Figure 1 Immunoperoxidase staining of the conjunctiva of a patient with OCP using anti-MI MAb and haematein counterstaining. The number of goblet cells is markedly decreased, but all goblet cells (arrows) and no epithelial cells were stained with the Mab (magnification $\times 500$ )

OCP patients, goblet cells were present in 16 of 22 conjunctival specimens, and less than six goblet cells were counted in the sections of 12 of them (fig 1). However, their cytoplasm expressed the same intense staining with anti-Ml mucin Mabs as the goblet cells of normal conjunctiva without any link between the presence of Ml antigen and Lewis or Secretor phenotypes.

Epithelial cells

Epithelial cells from OCP and normal conjunctivas were not stained with anti-Ml mucin Mabs.

\section{Lewis related epitopes (table 2)}

In OCP patients, Lewis related epitopes were exclusively expressed in the conjunctiva of Lewis positive individuals. $\mathrm{Le}^{\mathrm{a}}, \mathrm{Le}^{\mathrm{b}}$, and $\mathrm{Le}^{\mathrm{c}}$ phenotypes were found in 13, seven, and two patients, respectively. Sialyl $\mathrm{Le}^{\mathrm{a}}$ epitopes were expressed in goblet cells and epithelial cells of both $\mathrm{Le}^{\mathrm{a}}$ and $\mathrm{Le}^{\mathrm{b}}$ individuals as previously described in normal conjunctiva. ${ }^{18}$

\section{Goblet cells}

Of the 16 OCP conjunctival samples containing goblet cells, five expressed the $\mathrm{Le}^{\mathrm{a}}$ antigen and 11 the $\mathrm{Le}^{\mathrm{b}}$ antigen. The intensity of staining with anti-Le ${ }^{\mathrm{a}}$, anti-sialyl $\mathrm{Le}^{\mathrm{a}}$, and anti$\mathrm{Le}^{\mathrm{b}}$ Mabs was weaker in OCP than in controls. Sialyl $\mathrm{Le}^{\mathrm{a}}$ epitope immunostaining was negative or very weak in the goblet cells of two $\mathrm{Le}^{\mathrm{a}}$ and nine $\mathrm{Le}^{\mathrm{b}}$ OCP patients. One $\mathrm{Le}^{\mathrm{a}}$ and nine $\mathrm{Le}^{\mathrm{b}}$ patients did not express the $\mathrm{Le}^{\mathrm{a}}$ epitope in contrast with normal conjunctiva (according to the well known slight cross reaction of $\mathrm{Le}^{\mathrm{b}}$ and $\mathrm{Le}^{\mathrm{a}}$ - see above in "Monoclonal antibodies").

\section{Epithelial cells}

As in goblet cells, conjunctival epithelial cell immunoreactivity for sialyl $\mathrm{Le}^{\mathrm{a}}$ epitopes was slightly weaker in $\mathrm{Le}^{\mathrm{b}}$ OCP patients than in $\mathrm{Le}^{\mathrm{b}}$ normal individuals (fig 2). In two patients, no immunostaining was seen with the four Mabs
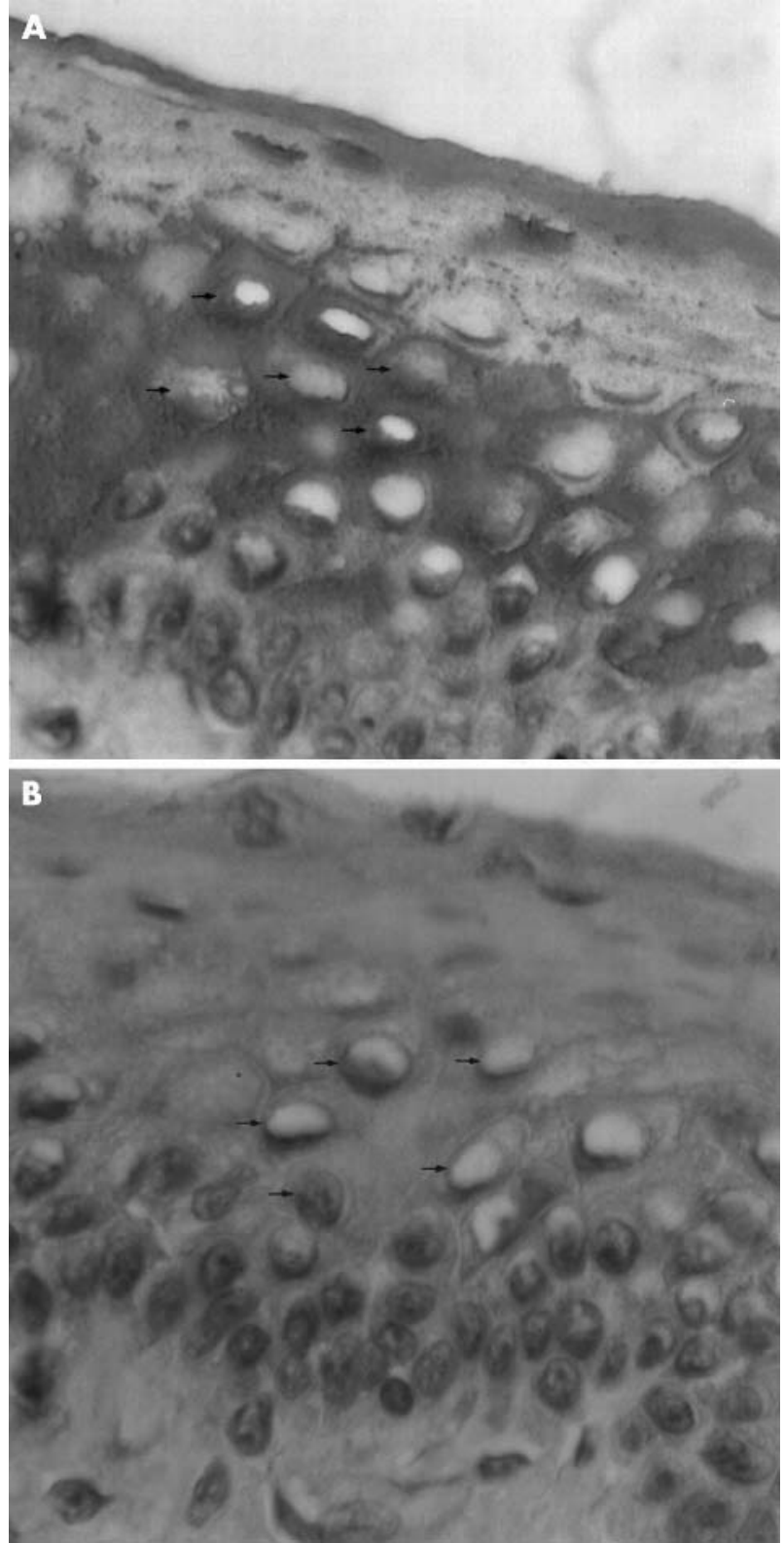

Figure 2 Conjunctival sample from a Le ${ }^{b}$ patient $(L e(a-b+))$ with OCP (A) Anti-Le $\mathrm{b}^{\mathrm{b}}$ Mab markedly stained the cytoplasm of the epithelial cells. (B) Anti-sialyl Le $\mathrm{a}$ Mab did not stain the epithelial cells. Small arrows indicate the nuclei of the haematein counterstained epithelial cells. Goblet cells are absent (magnification $\times 500$ ).

indicating that they had a $\mathrm{Le}^{\mathrm{c}}$ phenotype. $\mathrm{Le}^{\mathrm{d}}$ was not expressed in OCP patients

Comparison of the Lewis and secretor phenotypes of OCP patients and normal French population

Serological tests were performed in 17 of 22 OCP patients, and as in normal individuals, the same Lewis phenotype was found in red blood cells as in the conjunctiva. Therefore, immunohistological processing of the conjunctiva can be used for phenotypic studies comparing normal and cicatricial pemphigoid populations. There was a statistically significant difference $(\mathrm{p}=0.047)$ between both populations with respect to the non-secretor phenotype ( $\mathrm{Le}^{\mathrm{a}}$ and $\mathrm{Le}^{\mathrm{c}}$ ), which was found in $41 \%$ (nine out of 22 patients) and 20\% (17 out of 89 normal subjects) in the OCP group and the normal French population, ${ }^{18}$ respectively (table 2 ). 


\section{DISCUSSION}

According to the results of previous conventional histochemical studies, the mucin produced by goblet cells in OCP does not seem to differ from the mucin secreted by normal conjunctiva. Wells et al showed that goblet cells of OCP patients and normal individuals were stained with PNA (peanut agglutinin), HPA (Helix ponatia agglutinin), WGA (wheat germ agglutinin), and sWGA (succinylated wheat germ agglutinin). ${ }^{17}$ The binding of native WGA to the superficial conjunctival cells indicated the presence of $\mathrm{N}$-acetyl-glucosamine and/or sialic acid and its residues. Results of sodium dodecyl sulfate polyacrylamide gel electrophoresis studies have also shown that conjunctival mucus electrophoretic motility was similar in OCP, StevensJohnson syndrome, rosacea blepharoconjunctivitis, and normal individuals. ${ }^{16}$

As in previous studies, we showed that conjunctival goblet cells were scarce or absent in OCP patients. ${ }^{24-26}$ However, changes observed during OCP seem to be both quantitative and qualitative regarding mucin characteristics. The changes involved the carbohydrate structure of the oligosaccharide chains, but not the peptidic core encoded by MUC5AC gene. It should be of great interest to study membrane associated mucins which constitute a very important mucous component involved in many ocular surface disease. ${ }^{27}$ The immunoreactivity of blood group related antigens of goblet cells was reduced in OCP patients compared to normal individuals, suggesting a decreased activity of the fucosyl and sialyltransferases. ${ }^{28}$ Our finding of a weak sialyl $\mathrm{Le}^{\mathrm{a}}$ immunoreactivity in secretor patients suggests that sialylated glycoconjugates were less expressed. This may be partly the result of the competition between sialyltransferase and fucosyltransferase in secretor individuals. One hypothesis is that inflammation enhances the fixation of fucose rather than sialic acid. We showed a similar immunopathological pattern in pterygium-for example, a decreased sialyl Le ${ }^{a}$ immunostaining that may be related to a lower expression of ST3GalIII gene than in normal conjunctiva. ${ }^{28-30}$ Abnormal sialyltransferase activity observed during cell differentiation or maturation, particularly in tumours deserves to be investigated..$^{30}$ Decreased activity of glycosyl transferase could be assessed with direct assay of glycosyltransferase activity and/or measurement of mRNA levels in OCP patients.

We also demonstrated in our study that the percentage of individuals with non-secretor phenotype was higher in OCP patients than in the normal French population. The mechanisms by which oligosaccharide epitopes, and more particularly blood group related antigens, could be involved in the pathogenesis of OCP are unclear. Lewis, secretor, and $\mathrm{ABO}$ loci control the glycosyltransferase involved in the synthesis of the oligosaccharide chains. Their role has already been established in lung function, wheezing and asthma. ${ }^{31}$ It has been suggested that non-secretor patients could be more prone to develop mucous membrane diseases. ${ }^{29}$ It has been hypothesised that oligosaccharide epitopes are necessary for the recognition of some micro-organisms. Blood group related antigens have been involved in the pathogenesis of Helicobacter pylori associated diseases, as $\mathrm{Le}^{\mathrm{b}}$ patients may be more susceptible to develop gastric ulcer. ${ }^{32}{ }^{33}$

Six of the 22 OCP patients included in our study did not disclose the typical immunopathological features of the disease consisting of immune deposits at the conjunctival basement membrane. However, it has been shown that failure to demonstrate this deposition in a typical disease was possible, probably because of a lack of sensitivity of the technique. $^{34}$ The immunopathological proof is thereby not mandatory if the disease fullfils all diagnostic clinical criteria. In our study, all six OCP suspects had or later developed typical extraocular lesions of $\mathrm{CP}$, and responded well to systemic immunosuppressive therapy. Other systemic and ocular non-autoimmune fibrosing conditions were also easily ruled out. ${ }^{34}$ Moreover, there were no change in the conjunctival staining between biopsy proved and the suspect OCP patients.

In conclusion, we characterised the conjunctiva of patients with OCP with respect to the peptidic MUC5AC and blood group related antigens of mucins. We observed some patterns of glycoconjugate epitopes but not of the peptidic core detected by anti-Ml/MUC5AC MAb suggesting an abnormal glycosyltransferase expression. We also found an increase in the percentage of non-secretor phenotype in our patients. However, we are aware of the possible bias owing to the retrospective nature of the study as well as the lack of membrane associated mucin characterisation. Further studies including more patients are required to confirm these findings and to help us in a better understanding of the pathogenesis of autoimmune cicatrising conjunctivitis.

\section{Authors' affiliations}

C Creuzot-Garcher, A M Bron, Service d'Ophtalmologie, Université de Bourgogne, Dijon, France

T H Xuan, H Robin, Service d'Ophtalmologie, Fondation

Ophtalmologique A de Rothschild, Paris, France

P d'Athis, Service d'Informatique Médicale, Université de Bourgogne, Dijon, France

J Bara, U-482 INSERM, Hôpital Saint-Antoine, Paris, France

Presented in part at the annual meeting of the Association for Research in Vision and Ophthalmology, Ft Lauderdale, FL, USA, May 1999.

Correspondence to: C Creuzot-Garcher, Department of Ophthalmology, General Hospital, 3 rue du Faubourg Raines, 21000 Dijon, France; catherine.creuzot-garcher@chu-dijon.fr

Accepted for publication 29 February 2004

\section{REFERENCES}

1 Mondino BJ, Brown SI. Ocular cicatricial pemphigoid. Ophthalmology 1981;88:95-100.

2 Wright P. Cicatrizing conjunctivitis. Trans Ophthalmol Soc UK, 1986;105:1-17.

3 Foster CS. Cicatricial pemphigoid. Trans Am Ophthalmol Soc 1986:84:527-663.

4 Hoang-Xuan T, Robin H, Demers PE, et al. Pure ocular cicatricial pemphigoid. A distinct immunopathologic subset of cicatricial pemphigoid. Ophthalmology 1999; 106:355-61.

5 Rice BA, Foster CS. Immunopathology of cicatricial pemphigoid affecting the conjunctiva. Ophthalmology 1990;97:1476-83.

6 Hoang-Xuan T, Robin H, Heller M, et al. Epidermolysis bullosa acquisita diagnosed by direct immunoelectron microscopy of the conjunctiva. Ophthalmology 1997; 104:1414-20.

7 Carlstedt I, Lindgren $\mathrm{H}$, Sheehan JK. The macromolecular structure of the human cervical-mucus glycoproteins. Biochem J 1983;213:427-35.

8 Escande F, Aubert JP, Porchet N, et al. Human mucin gene MUC5AC: organization of its $5^{\prime}$-region and central repetitive region. Biochem $J$ 2001; 15:763-72.

9 Good AH, Yau O, Lamontagne LR, et al. Serological and chemical specificities of twelve monoclonal anti-Le ${ }^{a}$ and anti-Le ${ }^{b}$ antibodies. Vox Sang 1992;62:180-9.

10 Mollicone R, Bara J, Le Pendu J, et al. Immunohistologic pattern of type 1 (Lea, $\left.L^{b}\right)$ and type $2(X, Y, H)$ blood group-related antigens in the human pyloric and duodenal mucosae. Lab Invest 1985;53:219-27.

11 Bara J, Gautier R, Mouradian P, et al. Oncofetal mucin M1 epitope family: characterization and expression during colonic carcinogenesis. Int J Cancer 1991;47:304-10.

12 Bara J, Chastre E, Mahiou J, et al. Gastric M1 mucin, an early oncofoetal marker of colon carcinogenesis, is encoded by the MUC5AC gene. Int J Cancer 1998;75:767-73

13 Bara J, Nollet S, Forgue-Lafitte M-E, et al. Mapping of SOMU1 and M1 epitopes on the MUC5AC apomucin, an early marker of pancreas and colon carcinogenesis. Tumor Biol 2002;23:25.

14 Nollet S, Forgue-Lafitte M-E, Kirkham P, et al. Mapping of two epitopes on the apomucin encoded by MUC5AC gene: expression in normal $G$ tract and colon tumors. Int J Cancer 2002;99:336-43

15 Danjo $\mathrm{Y}$, Watanabe $\mathrm{H}$, Tisdale AS, et al. Alteration of mucin in human conjunctival epithelia in dry eye. Invest Ophthalmol Vis Sci 1998;39:2602-9.

16 Wells PA, Ashur ML, Foster CS. SDS-polyacrylamide gel electrophoresis of individual ocular mucus samples from patients with normal and diseased conjunctiva. Curr Eye Res 1986;5:823-31. 
17 Wells PA, De Siena-Shaw C, Rice B, et al. Detection of ocular mucus in normal human conjunctiva and conjunctiva from patients with cicatricial pemphigoid using lectin probes and histochemical techniques. Exp Eye Res 1988;46:485-97.

18 Garcher C, Bara J, Bron A, et al. Expression of mucin peptide and blood group $\mathrm{ABH}$ - and Lewis-related carbohydrate antigens in normal human conjunctiva. Invest Ophthalmol Vis Sci 1994;35:1184-91.

19 Mollicone R, Cailleau A, Imberty A, et al. Recognition of the blood group $\mathrm{H}$ type 2 trisaccharide epitope by 28 monoclonal antibodies and three lectins. Glycoconj J 1996;13:263-71.

20 Daher N, Bara J, Moubarak MD. Non A non B and Lewis related antigens in normal human prostates: an immuno-histochemical study of 20 antiglycoconjugate monoclonal antibodies. Blood Transf Immunohaematol 1987;30:681-4.

21 Bara J, Daher N, Mollicone R, et al. Immunohistological pattern of 20 monoclonal antibodies against non-A, non-B glycoconjugates in normal pyloric and duodenal mucosae. Rev Fr Transfus Immunohematol 1987;30:685-92.

22 Bara J, Gautier R, Le Pendu J, et al. Immunochemical characterization of mucins. Polypeptide (M1) and polysaccharide (A and Le ${ }^{\text {b) }}$ antigens. Biochem J 1988;254:185-93.

23 Bara J, Herrero-Zabaleta E, Mollicone R, et al. Distribution of GICA in normal gastrointestinal and endocervical mucosae and in mucinous ovarian cysts using antibody NS 19-9. Am J Clin Pathol 1986;85:152-9.

24 Bernauer W, Wright $\mathrm{P}$, Dart JK, et al. The conjunctiva in acute and chronic mucous membrane pemphigoid. An immunohistochemical analysis. Ophthalmology 1993;100:339-46.
25 Nelson JD, Wright JC. Conjunctival goblet cells densities in ocular surface disease. Arch Ophthalmol 1984;102:1049-51.

26 Mondino BJ. Cicatricial pemphigoid and erythema multiforme. Ophthalmology 1990;97:939-52.

27 Argueso P, Gipson IK. Epithelial mucins of the ocular surface: structure, biosynthesis and function. Exp Eye Res 2001;73:281-9.

28 Creuzot-Garcher C, Guerzider V, Assem M, et al. Alteration of sialyl lewis epitope expression in pterygium. Invest Ophthalmol Vis Sci 1999;40:1631-36.

29 Harduin-Lepers A, Recchi MA, Delannoy P. 1994, the year of sialyltransferases. Glycobiology 1995;5:741-58.

30 Kono M, Ohyama Y, Lee YC, et al. Mouse beta-galactoside alpha 2,3 sialyltransferases: comparison of in vitro substrate specificities and tissue specific expression. Glycobiology 1997;7:469-79.

31 Kauffman F, Frete C, Pham Q-T, et al. Association of blood group-related antigens to $\mathrm{FEV}_{1}$, wheezing and asthma. Am J Respir Crit Care Med 1996; 153:76-82.

32 Boren T, Falk P, Roth KA, et al. Attachment of Helicobacter pylori to human gastric epithelium mediated by blood group antigens. Science 1993;262:1892-5.

33 Go MF. What are the host factors that place an individual at risk for Helicobacter pylori-associated disease. Gastroenterology 1997; 113:S15-20.

34 Foster CS. Cicatricial pemphigoid. Trans Am Ophthalmol Soc 1986;84:527-663. 